\title{
Assessment of female fertility in the general practice setting
}

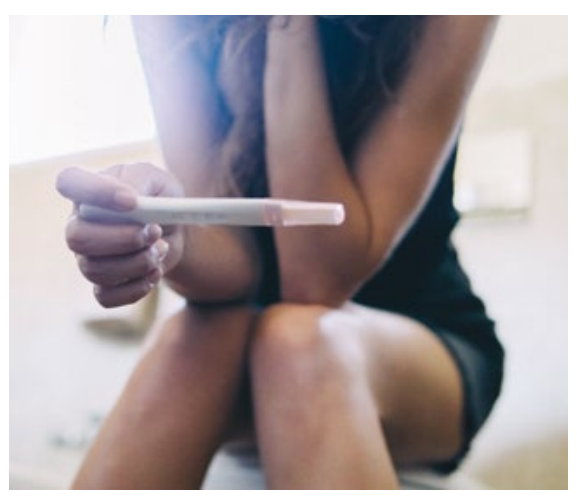

CPD

Sarah Hunt, Beverley Vollenhoven

\section{Background}

An assessment of female fertility may be undertaken in the general practice setting for a variety of reasons. These include concerns about future fertility when pregnancy is not immediately planned, a desire to consider elective oocyte cryopreservation and difficulty conceiving.

\section{Objective}

The aim of this article is to summarise indications, rationale and components of a comprehensive female fertility assessment in a primary care setting.

\section{Discussion}

The primary care physician has an essential role in providing women with guidance, counselling and assessment regarding fertility concerns. A complete initial assessment includes pre-pregnancy screening and counselling, and assessment of ovulation, ovarian reserve and pelvic anatomy to guide further investigation and management.
APPROXIMATELY $15 \%$ of couples will experience infertility. ${ }^{1}$ By definition, this is the failure to achieve pregnancy within 12 months of regular unprotected intercourse in a woman aged $<35$ years or within six months in a woman aged $>35$ years. $^{2}$

It is appropriate to perform an assessment of both male and female fertility factors at these junctures. Additionally, it would be prudent to undertake assessment in advance of these defined time points when there are factors on clinical assessment that could increase the risk of subfertility. These include oligomenorrhea; amenorrhoea; known or suspected uterine, tubal or peritoneal disease; severe endometriosis; multiple surgeries to the ovary or loss of one ovary; and previous chemotherapy or radiotherapy treatment. ${ }^{3,4}$ Known or suspected male subfertility should also prompt evaluation. ${ }^{4}$

The initial evaluation should comprise history-taking and examination focused on reproductive history factors and assessment of antenatal risk. A structured approach to initial reproductive history-taking is outlined in Box 1 . The examination should include an assessment of body mass index and blood pressure, a focused examination of the thyroid and breasts, and auscultation of the heart sounds. An abdominal and/or pelvic examination may be indicated on the basis of the presenting history and to complete cervical or infectious screening.

\section{Ovulation}

Ovulatory cycles are indicated by a history of regular cycle intervals of between 21 and 35 days with consistent characteristics and moliminal symptoms. Serum progesterone is not required to confirm ovulation when cycles are regular in frequency and character. If there is uncertainty, serum progesterone is confirmatory, provided that it is performed in the mid-luteal phase (ie seven days before the next expected menses). This may require serial progesterone levels to be performed for patients with minor inter-cycle variation. ${ }^{1}$

A clinical history consistent with anovulation requires further evaluation. A history of weight changes, stressors, hot flushes, visual disturbance, galactorrhoea or clinical hyperandrogenism may assist in delineating the cause of anovulation. Anovulation may be classified using the World Health Organization classification system (Table 1$).^{5-7}$ Accordingly, investigation of oligomenorrhoea or amenorrhoea should include folliclestimulating hormone (FSH), luteinising 
hormone and oestradiol (E2) in addition to thyroid stimulating hormone and prolactin concentrations. The recent international consensus guidelines for the assessment and management of polycystic ovarian syndrome (PCOS) endorse the Rotterdam criteria for diagnosis. ${ }^{8}$ Biochemical hyperandrogenism should be assessed using calculated free testosterone, free androgen index or calculated bioavailable testosterone using a liquid chromatography mass spectroscopy assay. Androstenedione and dehydroepiandrosterone may be considered for patients with normal testosterone. Given that PCOS is a diagnosis of exclusion, it is important to exclude non-classical congenital adrenal hyperplasia with a 17-hydroxyprogesterone concentration, which tests for the most common enzymatic deficiency in 21-hydroxylase. Exclusion of Cushing's disease also requires consideration, particularly when there are severe features of hyperandrogenism. ${ }^{8}$

\section{Ovarian reserve testing}

Both oocyte quantity and quality are important factors for successful conception.

Oocyte number peaks at 20 weeks' gestation; this is followed by a progressive decline that continues throughout reproductive life until a critical threshold is reached, resulting in menopause. ${ }^{9,10}$ Evaluating markers of ovarian reserve attempts to quantify the size of the remaining follicular pool as a surrogate for fertility. Routinely performed markers of ovarian reserve include day 2-4 FSH and $\mathrm{E} 2$, anti-Müllerian hormone (AMH) and assessment of antral follicle count (AFC) on transvaginal ultrasounds. ${ }^{10}$ No marker is directly able to measure the size of the primordial follicle pool. Ovarian reserve testing is an important component of an infertility assessment, and it provides information regarding the predicted ovarian response to assisted reproductive treatment (ART). However, caution should be exercised when performing the investigation outside this setting as it is not predictive of spontaneous conception and therefore interpretation is problematic.

A basal (day 2-4) FSH concentration is an indirect marker of ovarian reserve based on feedback inhibition of pituitary FSH secretion. In the case of diminished ovarian reserve, ovarian steroidogenesis will be insufficient to suppress early follicular FSH. A simultaneous measurement of serum E2 concentration should be performed, as this increases the sensitivity of FSH testing to assess ovarian reserve. Elevation in basal $\mathrm{E} 2$ concentration occurs in diminished ovarian reserve secondary to increased early follicular FSH but results in suppression of pituitary FSH secretion and an FSH concentration

\section{Box 1. Systematic approach to female reproductive history}

\section{Duration of infertility}

- Frequency and timing of intercourse

- Sexual dysfunction

\section{Gynaecological and obstetric past history}

- Cervical screening results, previous treatments

- Previous pregnancies: time to conceive, management of early pregnancy loss and termination, mode and timing of delivery and antenatal, intrapartum or post-partum complications

- Pelvic infection

- Menstrual history: cycle interval, duration of bleeding and associated abnormal uterine bleeding and dysmenorrhoea

- Dyspareunia

Previous medical history

- Medical comorbidities: management and stability

- Previous surgery

Medications, including any allergies

- Past and present medication use

- Previous contraceptive use

- Vaccination history: rubella, varicella, hepatitis B, influenza

- Folic acid and iodine supplementation

Family history

- Heritable conditions

- Premature ovarian insufficiency

Social history

- Smoking, alcohol and recreational drug use

\section{Table 1. World Health Organization classification of anovulation ${ }^{5-7}$}

\begin{tabular}{lll}
\hline Group & Classification & Causes \\
\hline Group 1 & $\begin{array}{l}\text { Hypogonadotropic } \\
\text { anovulation }\end{array}$ & $\begin{array}{l}\text { Hypothalamic amenorrhoea: stress, weight loss, } \\
\text { excessive exercise } \\
\text { Hyperprolactinaemia } \\
\end{array}$ \\
& & $\begin{array}{l}\text { Primary pituitary disease: infiltrative disease, central } \\
\text { nervous system tumour } \\
\text { Congenital gonadotropin-releasing hormone deficiency }\end{array}$ \\
\hline Group 2 & Normogonadotropic & Polycystic ovary syndrome \\
& anovulation & Congenital adrenal hyperplasia \\
\hline Group 3 & $\begin{array}{l}\text { Hypergonadotropic } \\
\text { anovulation }\end{array}$ & Premature ovarian insufficiency \\
& &
\end{tabular}


that falls within the normal range. An FSH concentration of $>10 \mathrm{IU} / \mathrm{L}$ has high specificity for poor ovarian response to controlled ovarian hyperstimulation in women undergoing ART ( $\leq 4$ oocytes retrieved at egg collection) but is poorly sensitive. Sensitivity is even poorer for predicting conception, and $\mathrm{FSH}$ demonstrates significant intra- and inter-cycle variation. Interpretation also requires intact function of the hypothalamic-pituitary-ovarian axis, which limits clinical application. ${ }^{10-12}$

$\mathrm{AMH}$ is produced by the granulosa cells of the pre-antral and antral follicles, thereby reflecting the size of the primordial follicle pool. ${ }^{13}$ Serum AMH concentration is inversely related to female age and shows minimal variation both within and between cycles. Therefore, testing on any day of the menstrual cycle is appropriate. ${ }^{10}$ Results will often be reported as the absolute number as well as the centile in relation to female age, which assists interpretation. Elevated AMH is predictive of an excessive response to controlled ovarian hyperstimulation during ART, while low AMH is predictive of an increased risk of poor ovarian response. ${ }^{14,15}$ For women attempting spontaneous conception, AMH correlates poorly with fecundity. ${ }^{16}$

AFC is the total number of follicles in both ovaries with a mean width measurement of 2-10 mm on ultrasound assessment. Although it is possible to count throughout the menstrual cycle, assessment in the early follicular phase avoids underestimation of follicle number due to the presence of a corpus luteum or large dominant follicle. Ultrasonography is an operator-dependent technique; therefore, AFC may vary between operators and be influenced by other imaging factors such as machine resolution and patient body habitus. Antral follicle number $>15$ or $<3-7$ predicts hyper response and poor ovarian response, respectively, but correlates poorly with pregnancy. ${ }^{15,17}$

\section{Pelvic anatomy and tubal patency}

Transvaginal pelvic ultrasonography is an important component of female fertility assessment. In addition to assessing the AFC, it allows for assessment of the uterine anatomy and structure and mobility of the pelvic organs.

Uterine polyps, fibroids and abnormalities of the Müllerian tract may be detected on transvaginal ultrasounds with or without saline infusion. Venetian blind shadowing, globular uterine appearance, loss of junctional zone definition and 'question mark sign' are all sonographic features associated with adenomyosis (Figure 1). ${ }^{18}$ Endometriosis may be diagnosed using ultrasonography in the hands of an experienced operator. Limitation in mobility of the adnexal structures and/or specific probe tenderness may indicate superficial endometriosis or other pelvic pathology associated with adhesion formation. Direct visualisation of nodules and endometriomas (Figure 2) and absent sliding of the rectum against the uterus are features of deep infiltrating disease. In addition to guiding a presumptive diagnosis, ultrasonography assists in surgical planning for women with deep infiltrating disease, particularly with regard to bowel involvement. ${ }^{19}$ Any of these findings may affect oocyte capture to the fallopian tube; fallopian tube structure, patency and transport; successful implantation and ongoing pregnancy.

Tubal patency may be assessed using hysterosalpingography, hysterosalpingocontrast sonography or hydrotubation at laparoscopy. Tubal patency testing may also be combined with a therapeutic intervention such as flushing with oil-based contrast, which may increase conception in the following six months when compared with flushing with water-based contrast. ${ }^{20,21} \mathrm{An}$ assessment of tubal patency is generally considered following preliminary fertility investigations (Box 2) including semen analysis, and the modality is decided on the basis of the suspected cause of infertility, concurrent symptoms, anticipated treatment and predicted risk of tubal disease.

\section{Preconception screening}

Women presenting for assessment should have serology performed for rubella and varicella immunoglobulin $G(\operatorname{Ig} G)$ at a

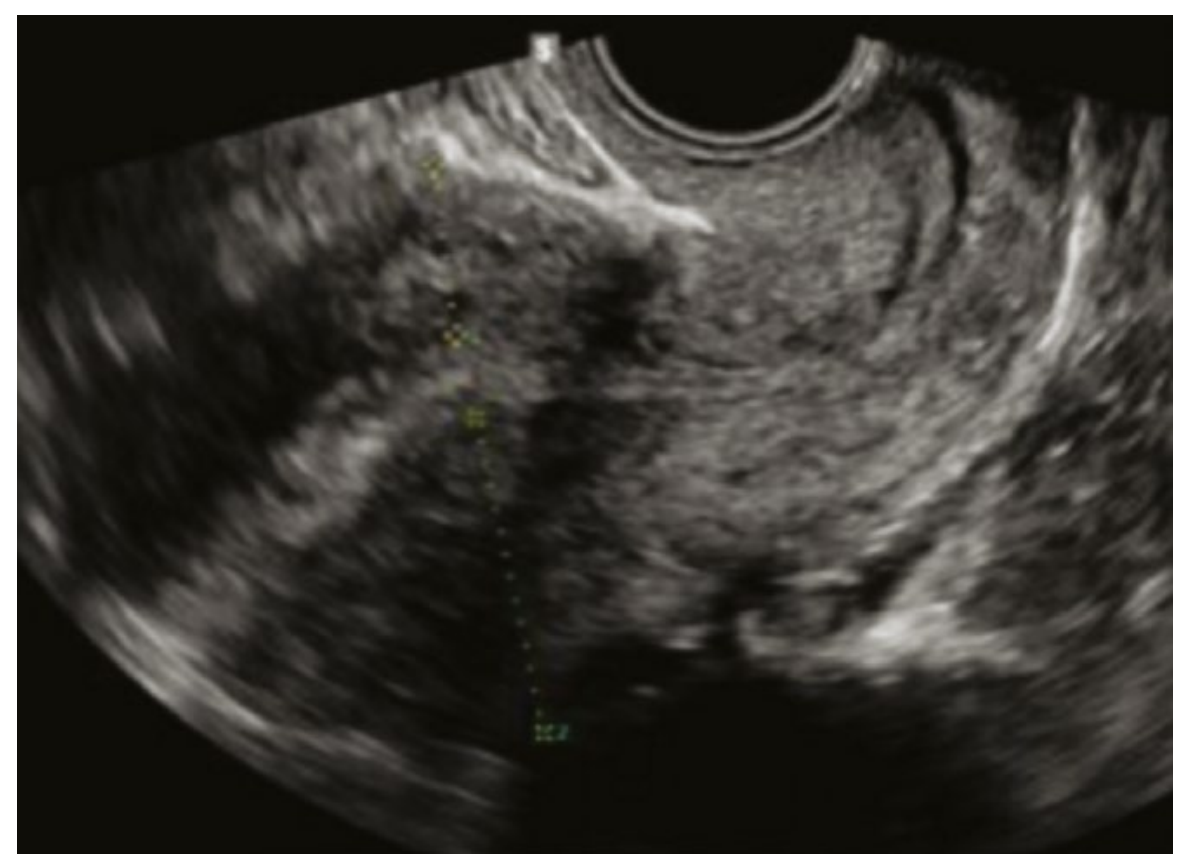

Figure 1. Pelvic ultrasound with features suggestive of adenomyosis: heterogeneous myometrium and 'venetian blind' artefact 
minimum. This allows for vaccination of susceptible women prior to pregnancy, although women should be told to defer conception for at least 28 days post-vaccination. ${ }^{22}$ Additionally, serology testing for hepatitis $\mathrm{B}$, hepatitis $\mathrm{C}$, human immunodeficiency virus and syphilis may be performed, as these form part of the routine antenatal investigations. Performing these tests prior to conception allows for appropriate pre-pregnancy counselling to take place and any infectious conditions to be optimised to reduce maternal and fetal risk. ${ }^{23}$

Genetic carrier screening should also be discussed and offered to all women contemplating pregnancy. ${ }^{24}$ Thalassaemia screening with a full blood examination is a component of routine antenatal screening. Further haemoglobin electrophoresis and DNA studies may be indicated on the basis of results (red cell indices), family history or high-risk ethnic group. Screening panels are now available to simultaneously test for carriership of common genetic conditions. Limited panels test for the three most common conditions: spinal muscular atrophy, cystic fibrosis and fragile $\mathrm{X}$ premutation. Extended panels offer testing for hundreds of genetic conditions, including both autosomal and $\mathrm{X}$-linked recessive inheritance, with a single test. Given the high likelihood of carriership detection for one or more conditions in any individual undertaking an extended panel, pre- and post-test counselling is essential, and the advantages and disadvantages of sequential or simultaneous screening of the couple should be discussed. ${ }^{25}$

\section{Assessment of fertility for women considering oocyte cryopreservation}

Age is the most important determinant of female fertility. In Australia, the average age of women at first birth in 2015 was 28.9 years, compared with 28.1 years in 2005 ; the proportion of births to mothers over the age of 35 years was $22 \% .{ }^{26}$ On a societal level, the shift towards delayed childbearing has led to a decrease in total fertility rate. ${ }^{27}$

Infertility is most prevalent in women aged $>35$ years and results in significant burden of disease. ${ }^{28}$

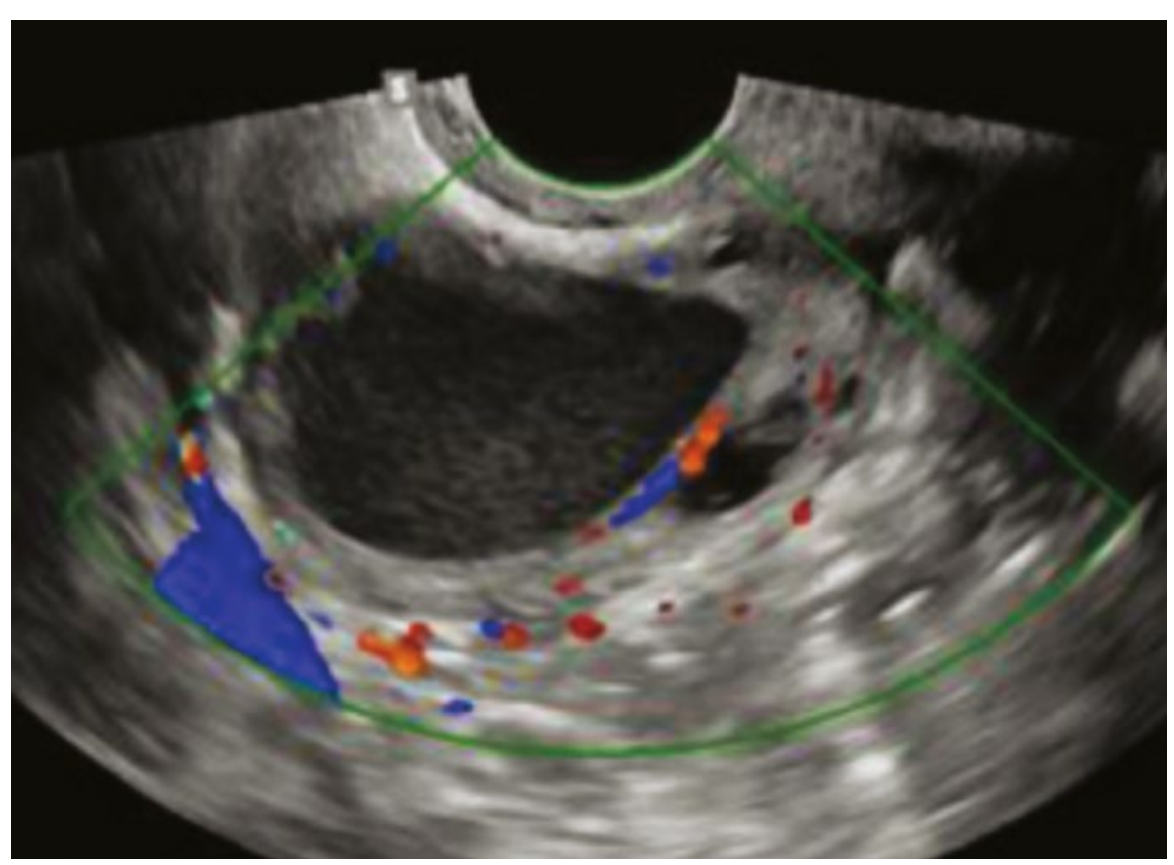

Figure 2. Pelvic ultrasound showing typical 'ground glass' appearance of an endometrioma

\section{Box 2. Preliminary fertility investigations}

Day 2-4 follicle-stimulating hormone, luteinising hormone, oestradiol

Anti-Müllerian hormone

Thyroid stimulating hormone

Transvaginal ultrasonography of the pelvis: antral follicle count, pelvic anatomy and features of deep infiltrating endometriosis

Blood group and antibody screen

Full blood examination

Rubella, varicella immunoglobulin $G$

Hepatitis $B$, hepatitis $C$, human immunodeficiency virus and syphilis serology

Genetic carrier screening if desired: thalassaemia, triple screen (fragile $X$ syndrome, cystic fibrosis, spinal muscular atrophy), extended carrier screen

The advent of vitrification technology means that cryopreserved oocytes have survival rates $>84 \%$ and comparable in vitro fertilisation outcomes to fresh oocytes. ${ }^{29}$ Increasingly, women are considering elective oocyte cryopreservation to allow an opportunity to conceive biologically related children at a later age. ${ }^{30}$

An appropriate preliminary assessment for a woman considering elective oocyte cryopreservation would include systematic history-taking and examination as outlined in Box 1 and investigations as outlined in Box 2. This provides valuable information for the assessment of medical comorbidities and forms the basis for counselling regarding the risks and benefits of elective oocyte cryopreservation and the expected response to controlled ovarian hyperstimulation. The ideal age to freeze oocytes is prior to 35 years of age, as poorer pregnancy outcomes related to deteriorating egg quality are achieved from oocytes vitrified after the age of 35 years. ${ }^{27}$

\section{Conclusion}

A comprehensive assessment of female fertility aims to determine underlying causes of subfertility, provide an opportunity for pre-pregnancy screening 
and counselling, and offer genetic screening options. Assessment of ovulatory function, ovarian reserve and pelvic anatomy provide a basis for further tailored investigations and infertility management.

\section{Authors}

Sarah Hunt MBBS, FRANZCOG, MRMed, PhD candidate, Monash University, Vic; Consultant Gynaecologist, Monash Health, Vic; CREI Clinical Fellow, Monash IVF, Vic

Beverley Vollenhoven PhD, FRANZCOG, CREI Deputy Head, Department of Obstetrics and Gynaecology, Monash University, Vic; Director of Gynaecology, Monash Health, Vic; Consultant Gynaecologist, Monash IVF, Vic. beverley.vollenhoven@monash.edu Competing interests: None.

Funding: None.

Provenance and peer review: Commissioned, externally peer reviewed.

\section{References}

1. Pfeifer S, Butts S, Dumesic D, et al. Diagnostic evaluation of the infertile female: A committee opinion. Fertil Steril 2015;103(6):e44-50. doi: 10.1016/j.fertnstert.2015.03.019.

2. Pfeifer S, Goldberg J, Lobo R, et al. Definitions of infertility and recurrent pregnancy loss: A committee opinion. Fertil Steril 2013;99(1):63. doi: 10.1016/j.fertnstert.2012.09.023.

3. Hunt S, Vollenhoven B. Fertility preservation in women with cancer and afterward. Climacteric 2019;22(6):579-83. doi: 10.1080/13697137.2019.1607285.

4. Infertility workup for the women's health specialist: ACOG committee opinion, number 781. Obstet Gynecol 2019;133(6):e377-e384. doi: 10.1097/AOG.0000000000003271.

5. Advances in methods of fertility regulation: Report of a WHO scientific group. World Health Organ Tech Rep Ser 1973;527:1-42.

6. Baird DT, Balen A, Escobar-Morreale HF, et al. Health and fertility in World Health Organization group 2 anovulatory women. Hum Reprod Update 2012;18(5):586-99. doi: 10.1093/humupd/dms019.

7. Dhont M. WHO-classification of anovulation: Background, evidence and problems. International Congress Series 2005;1279:3-9. doi: 10.1016/j. ics.2004.12.028.

8. Teede HJ, Misso ML, Boyle JA, et al. Translation and implementation of the Australian-led PCOS guideline: Clinical summary and translation resources from the international evidence-based guideline for the assessment and management of polycystic ovary syndrome. Med J Aust 2018;209(S7):S3-S8.

9. Vollenhoven B, Hunt S. Ovarian ageing and the impact on female fertility. F1000Res 2018;7. doi: 10.12688/f1000research.16509.1.

10. Tal R, Seifer DB. Ovarian reserve testing: $A$ user's guide. Am J Obstet Gynecol 2017;217(2):129-40. doi: 10.1016/j.ajog.2017.02.027.

11. Broekmans FJ, Kwee J, Hendriks DJ, Mol BW, Lambalk CB. A systematic review of tests predicting ovarian reserve and IVF outcome. Hum Reprod Update 2006;12(6):685-718. doi: 10.1093/ humupd/dml034
12. Esposito MA, Coutifaris C, Barnhart KT. A moderately elevated day $3 \mathrm{FSH}$ concentration has limited predictive value, especially in younger women. Hum Reprod 2002;17(1):118-23. doi: 10.1093/humrep/17.1.118.

13. Weenen C, Laven JSE, Von Bergh ARM, et al. Anti-Müllerian hormone expression pattern in the human ovary: Potential implications for initial and cyclic follicle recruitment. Mol Hum Reprod 2004;10(2):77-83. doi: 10.1093/molehr/gah015.

14. La Marca A, Sighinolfi G, Radi D, et al. AntiMüllerian hormone (AMH) as a predictive marker in Assisted Reproductive Technology (ART). Hum Reprod Update 2010;16(2):113-30. doi: 10.1093/ humupd/dmp036.

15. Broer SL, van Disseldorp J, Broeze KA, et al. Added value of ovarian reserve testing on patient characteristics in the prediction of ovarian response and ongoing pregnancy: An individual patient data approach. Hum Reprod Update 2013;19(1):26-36. doi: 10.1093/humupd/dms041.

16. Steiner AZ, Pritchard D, Stanczyk FZ, et al. Association between biomarkers of ovarian reserve and infertility among older women of reproductive age. JAMA 2017;318(14):1367-76. doi: 10.1001/jama.2017.14588.

17. Nastri CO, Teixeira DM, Moroni RM, Leitão VMS, Martins WP. Ovarian hyperstimulation syndrome: Pathophysiology, staging, prediction and prevention. Ultrasound Obstet Gynecol 2015;45(4):377-93. doi: 10.1002/uog.14684.

18. Andres MP, Borrelli GM, Ribeiro J, Baracat EC, Abrão MS, Kho RM. Transvaginal ultrasound for the diagnosis of adenomyosis: Systematic review and meta-analysis. J Minim Invasive Gynecol 2018;25(2):257-64. doi: 10.1016/j.jmig.2017.08.653.

19. Van den Bosch T, Van Schoubroeck D. Ultrasound diagnosis of endometriosis and adenomyosis: State of the art. Best Pract Res Clin Obstet Gynaecol 2018;51:16-24. doi: 10.1016/j. bpobgyn.2018.01.013.

20. Dreyer K, van Rijswijk J, Mijatovic V, et al. Oil-based or water-based contrast for hysterosalpingography in infertile women. N Engl J Med 2017;376(21):2043-52. doi: 10.1056/ NEJMoa1612337.

21. Wang R, van Welie N, van Rijswijk J, et al. Effectiveness on fertility outcome of tubal flushing with different contrast media: Systematic review and network meta-analysis. Ultrasound Obstet Gynecol 2019;54(2):172-81. doi: 10.1002/ uog. 20238 .

22. Australian Technical Advisory Group on Immunisation. Australian immunisation handbook. Canberra: DoH, 2018.

23. The Royal Australian and New Zealand College of Obstetricians and Gynaecologists. Routine antenatal assessment in the absence of pregnancy complications. Melbourne, Vic: RANZCOG, 2019.

24. The Royal Australian and New Zealand College of Obstetricians and Gynaecologists. Genetic carrier screening. Melbourne, Vic: RANZCOG, 2019.

25. Henneman L, Borry P, Chokoshvili D, et al. Responsible implementation of expanded carrier screening. Eur J Hum Genet 2016;24(6):e1-e12.

26. Australian Institute of Health and Welfare. Australia's mothers and babies 2015 - In brief. Perinatal statistics series no. 33. Cat no. PER 91. Canberra: AlHW, 2017.

27. Chambers GM, Wand $H$, Macaldowie A, et al. Population trends and live birth rates associated with common ART treatment strategies. Hum Reprod 2016;31(11):2632-41. doi: 10.1093/humrep/ dew232.
28. Lew R, Foo J, Kroon B, Boothroyd C, Chapman M. ANZSREI consensus statement on elective oocyte cryopreservation. Aust N Z J Obstet Gynaecol 2019;59(5):616-26. doi: 10.1111/ajo.13028.

29. Argyle CE, Harper JC, Davies MC. Oocyte cryopreservation: Where are we now? Hum Reprod Update 2016;22(4):440-49. doi: 10.1093/ humupd/dmw007.

30. Goldman KN, Grifo JA. Elective oocyte cryopreservation for deferred childbearing. Curr Opin Endocrinol Diabetes Obes 2016;23(6):458-64. doi: 10.1097/MED.0000000000000291. 